\title{
Global versus local statement of stress constraints in topology optimization of continuum structures
}

\author{
J. París, F. Navarrina, I. Colominas \& M. Casteleiro \\ GMNI, Group of Numerical Methods in Engineering, \\ Department of Applied Mathematics, Universidad de A Coruña, \\ E.T.S. de Ingenieros de Caminos, Canales y Puertos, \\ A Coruña, Spain
}

\begin{abstract}
Structural topology optimization problems have been traditionally set out in terms of maximum stiffness formulations. In this approach, the goal is to distribute a given amount of material in a certain region, so that the stiffness of the resulting structure is maximized for a given load case. Even though this approach is quite convenient, it also entails some serious conceptual and practical drawbacks.

The authors, in common with other research groups, have been working for a few years on the possibility of stating these kinds of problems by means of a FEMbased minimum weight with stress (and/or displacement) constraints formulation. The physical meaning of this approach is closer to the engineering point of view. Furthermore, most of the above mentioned drawbacks could be removed this way. However, this also leads to more complicated optimization problems with much higher computational requirements, since a large number of highly non-linear (local) constraints must be taken into account to limit the maximum stress (and/or displacement) at the element level. In this paper, we explore the feasibility of defining a so-called global constraint, whose basic aim is to limit the maximum stress (and/or displacement) simultaneously within all the structure by means of one single inequality. Should this global constraint perform adequately, the complexity of the underlying mathematical programming problem should be drastically reduced. Finally, we compare the results provided by both types of constraints in some application examples.
\end{abstract}




\section{Introduction}

Structural topology optimization problems have been traditionally set out in terms of maximum stiffness (minimum compliance) formulations. In this approach, the goal is to distribute a given amount of material in a certain region, so that the stiffness of the resulting structure is maximized (the compliance, or energy of deformation, is minimized) for a given load case [1,2]. Even though this approach is quite convenient, it also entails some serious drawbacks, mainly: multiple load cases can not be considered; self-weight is normally ignored; the result varies with the amount of material to be distributed; and the final design could be unfeasible in practice, since no constraints are imposed on stresses (and/or displacements). Moreover, the maximum stiffness problem is essentially ill-posed. Thus, the solution oscillates as the discretization refinement is increased, what gives raise to mesh-dependent checkerboard layouts. This difficulty can be partially overcome by introducing porous materials [1]. But, on a regular basis, a spread porous material distribution is considered an unwanted result. Hence, additional penalization and stabilization techniques and image filters must be employed to avoid numerical instabilities and unrealistic -or simply useless- final solutions.

The authors, as other research groups, are working since a few years in the possibility of stating this kind of problems by means of a FEM-based minimum weight with stress (and/or displacement) constraints approach. Obviously, the physical meaning of this approach is closer to the engineering point of view, while any kind of constraint under multiple load cases could also be considered.

The basic and most intuitive procedure to preclude excessively high stresses (and/or excessively large displacements) within all the structure consists in limiting the maximum stress (and/or displacement) at a series of given points within each element $[3,4]$. This is commonly referred to as the "local (statement of) constraints approach". Thus, one can easily state quite complete and realistic optimization problems. The optimized solutions seem to be correct from the engineering point of view and their appearance could be considered closer to the engineering intuition than the results provided by the maximum stiffness approach. Furthermore, neither stabilization techniques nor image filters seem to be necessary to preclude unwanted final results [4]. However, this also leads to more complicated optimization problems with much higher computational requirements, since a large number of highly non-linear (local) constraints must be taken into account to limit the maximum stress (and/or displacement) at the element level.

In this paper, we explore the feasibility of defining a so-called global constraint, which basic aim is to limit the maximum stress (and/or displacement) simultaneously within all the structure by means of one single inequality. This is commonly referred to as the "global (statement of) constraints approach". Should this global constraint perform adequately, the complexity of the underlying mathematical programming problem should be drastically reduced. The global constraint formulation that we present hereafter is based on the Kreisselmeier-Steinhauser function [5]. Finally, we compare the results obtained by means of both the local and the global statement of constraints in some application examples. 


\section{The optimization problem}

In terms of a FEM-based minimum weight with stress (and/or displacement) constraints formulation, the topology optimization problem can be written as [4]

$$
\begin{array}{lll}
\text { Find } & \boldsymbol{\rho}=\left\{\boldsymbol{\rho}_{e}\right\}, & e=1, \ldots, N_{e} \\
\text { that minimizes } & F(\boldsymbol{\rho}) & \\
\text { verifying } & g_{j}(\boldsymbol{\rho}) \leq 0, & j=1, \ldots, m \\
& 0<\rho_{\min } \leq \rho_{e} \leq 1, & e=1, \ldots, N_{e}
\end{array}
$$

where the design variable $\rho_{e}$ is the relative density of element number $e$, what is assumed constant within the element. Thus, if $d \Omega$ is the volume of a differential region within element number $e$, the volume occupied by the porous material within the differential region will be $\rho_{e} d \Omega$. The lower limit for the relative density $\left(\rho_{\text {min }}\right)$ is introduced to preclude the entire hollowing out of the elements (since the concepts of displacement, strain and stress become meaningless and the stiffness matrix could even be singular in such a case).

The objective function is defined as [4]

$$
F(\boldsymbol{\rho})=\sum_{e=1}^{N_{e}}\left(\rho_{e}\right)^{\frac{1}{p}} \int_{\Omega_{e}} \gamma_{m a t} d \Omega
$$

where $\Omega_{e}$ is the element number $e, \gamma_{\text {mat }}$ is the density of the material (assumed constant), and $p \geq 1$ is a tuning parameter that can be adjusted to favor a mainly compact distribution of material (since the intermediate values of the relative density are increasingly penalized as the value of $p$ grows).

It seems quite obvious that any kind of constraint could be taken into account in the above stated optimization problem. For the seek of simplicity, further discussion and examples are restricted to considering stress constraints type

$$
\widehat{\sigma}_{\text {min }} \leq \widehat{\sigma}\left(\boldsymbol{\sigma}_{j}^{h}(\boldsymbol{\rho})\right) \quad \text { and/or } \quad \widehat{\sigma}\left(\boldsymbol{\sigma}_{j}^{h}(\boldsymbol{\rho})\right) \leq \widehat{\sigma}_{\max }
$$

where $\boldsymbol{\sigma}_{j}^{h}(\boldsymbol{\rho})$ are the FEM-computed components of the stress tensor at each given point $P_{j}$ for the actual values of the relative densities $\rho$. The details on the FEM formulation for the structural analysis problem with relative density can be found in [4]. Finally, $\widehat{\sigma}(\boldsymbol{\sigma})$ is the reference stress expression that corresponds to the failure criteria being used (which values are limited).

In the $2 \mathrm{D}$ examples presented in this paper we consider materials with equal tensile and compressive strength limits. Thus, $\widehat{\sigma}(\boldsymbol{\sigma})$ is the Von Mises reference stress expression and $\widehat{\sigma}_{\max }$ is the elastic stress limit of the material [4]. Then the constraints considered in (1) can be written as

$$
g_{j}(\boldsymbol{\rho})=\widehat{\sigma}\left(\boldsymbol{\sigma}_{j}^{h}(\boldsymbol{\rho})\right)-\widehat{\sigma}_{\max } \leq 0 .
$$




\subsection{Local statement of constraints}

Without losing generality, let's suppose that one stress constraint is imposed at one given point per element. Then, the optimization problem takes the form

$\begin{array}{lll}\text { Find } & \boldsymbol{\rho}=\left\{\boldsymbol{\rho}_{e}\right\}, & e=1, \ldots, N_{e} \\ \text { that minimizes } & F(\boldsymbol{\rho}) & \\ \text { verifying } & g_{e}(\boldsymbol{\rho}) \leq 0, & e=1, \ldots, N_{e} \\ & 0<\rho_{\min } \leq \rho_{e} \leq 1, & e=1, \ldots, N_{e}\end{array}$

This is commonly referred to as the "local (statement of) constraints approach".

However, stress constraints type (4) can exhibit the so-called "singularity phenomena", that is due to the discontinuous nature of the stress when the relative density tends to zero [6]. Briefly, reaching the optimum could call for removing all the material within a certain element $\Omega_{e}$. However, the corresponding restriction type (4) could be more severely violated as we get closer to the optimum (that is, for decreasing values of $\rho_{e}$ slightly greater than 0 ), since the stress could rise as the material is being removed (until the element is completely hollowed out). Under these conditions, the gradient of the constraint would be negative in the vicinity of the optimum. Thus, any consistent non linear programming algorithm would try to increase the relative density instead of reducing it, what precludes convergence to the exact solution of the problem [4]. Singularity phenomena have also been observed in some theoretical truss optimization problems [7] and in other fields of structural optimization [3]. For this reason, statements type (4) are not fully satisfactory and they must be rewritten some way. Following the ideas of several authors $[7,3,4]$ we propose the alternative statement for the local stress constraint

$$
\begin{aligned}
& g_{e}(\boldsymbol{\rho})=\left[\widehat{\sigma}\left(\boldsymbol{\sigma}_{e}^{h}(\boldsymbol{\rho})\right)-\widehat{\sigma}_{\max } \varphi_{e}\right]\left(\rho_{e}\right)^{q} \leq 0, \\
& \text { being } \varphi_{e}=1-\varepsilon+\frac{\varepsilon}{\rho_{e}}
\end{aligned}
$$

When $q=0$, limits are imposed on the stress. When $q=1$, limits are imposed on the so-called effective stress [4], what helps to remove some singularities. On the other hand, the value of the "relaxation parameter" $\varepsilon \in[0.001,0.1]$ must be reduced as we approach the optimum during the optimization process.

The solutions to problems type (5) with constraints type (6) seem to be correct from the engineering point of view and their appearance could be considered closer to the engineering intuition than the results provided by the maximum stiffness approach [4]. Furthermore, neither stabilization techniques nor image filters seem to be necessary to preclude unwanted final results. However, these optimization problems are much more complicated and they have much higher computational requirements than the ones emerging from the maximum stiffness approach, since we have to deal now with a large number of highly non-linear constraints type (6). 


\subsection{Global statement of constraints}

We explore now the feasibility of limiting the stress simultaneously within all the structure by means of one single inequality. Should this be possible, the optimization problem should reduce to

$$
\begin{array}{lll}
\text { Find } & \boldsymbol{\rho}=\left\{\boldsymbol{\rho}_{e}\right\}, \quad e=1, \ldots, N_{e} \\
\text { that minimizes } & F(\boldsymbol{\rho}) \\
\text { verifying } & G(\boldsymbol{\rho}) \leq 0, \\
& 0<\rho_{\text {min }} \leq \rho_{e} \leq 1, \quad e=1, \ldots, N_{e}
\end{array}
$$

This is commonly referred to as the "global (statement of) constraints approach". Obviously, if the so-called global constraint $G(\boldsymbol{\rho})$ performs adequately, the complexity of the mathematical programming problem and the computational requirements (both the data storage and the computing time) should be drastically reduced in comparison with (5).

Therefore, the essence is to define an adequate procedure for aggregating all the local constraints in a single global one. The global constraint formulation that we present hereafter is based on the Kreisselmeier-Steinhauser function, that is mainly being used at present in aero-structural optimization [5]. Furthermore, we have introduced some modifications that improve the numerical performance of the resulting global constraint. The proposed global constraint takes the form

$$
\begin{aligned}
& G_{K S}(\boldsymbol{\rho})=\left[\frac{1}{\mu} \ln \left(\sum_{e=1}^{N_{e}} e^{\mu\left(\widehat{\sigma}_{e}^{*}-1\right)}\right)-\frac{1}{\mu} \ln \left(N_{e}\right)\right] \leq 0 \\
& \text { being } \widehat{\sigma}_{e}^{*}=\frac{\widehat{\sigma}\left(\boldsymbol{\sigma}_{e}^{h}(\boldsymbol{\rho})\right)}{\widehat{\sigma}_{\max } \varphi_{e}} .
\end{aligned}
$$

The use of the normalized reference stress $\widehat{\sigma}_{e}^{*}$ is intended to rescale the arguments of the exponential terms. In addition, it helps to prevent a possible overflow condition to occur.

On the other hand, $\mu$ is a tuning parameter that penalizes the failure to satisfy the local constraints. In theory, global constraint (8) becomes equivalent to the corresponding whole set of local constraints when $\mu$ tends to infinity. However, when the value of $\mu$ is too large, global constraint (8) can become too difficult to manage, both for practical and theoretical reasons. Thus, for increasing values of $\mu$ (after a certain point) the expected accuracy of the sensitivity analysis decreases; in addition, the non-linearity of the global constraint function is boosted; and overflow conditions are more likely to occur. Consequently, it becomes more difficult to obtain a reasonably good numerical solution to problem (7). On the other hand, global constraint (8) will not adequately represent the corresponding whole set of local constraints if the value of $\mu$ is not large enough. In such a case, the solution to problem (7) will not be satisfactory. Therefore, it is extremely important to assign a correct value to the parameter $\mu$. 
Figures 1 and 2 depict the value of the global constraint for different values of the parameter $\mu$ in different conditions. In Figure $1, \widehat{\sigma}_{e}^{*}=0.90$ at $50 \%$ of the elements. The curves compare the values of the global constraint for different values of $\widehat{\sigma}_{e}^{*}$ (assumed all equal) at the remaining elements. In Figure $2, \widehat{\sigma}_{e}^{*}=$ 0.90 at the elements in which the corresponding local constraint is satisfied, and $\widehat{\sigma}_{e}^{*}=1.10$ at the elements in which the corresponding local constraint is violated. The curves compare the values of the global constraint for a growing percentage of violated local constraints. On a regular basis, it seems reasonable to adjust the value of $\mu$ between 20 and 30, or between 15 and 40 as much.

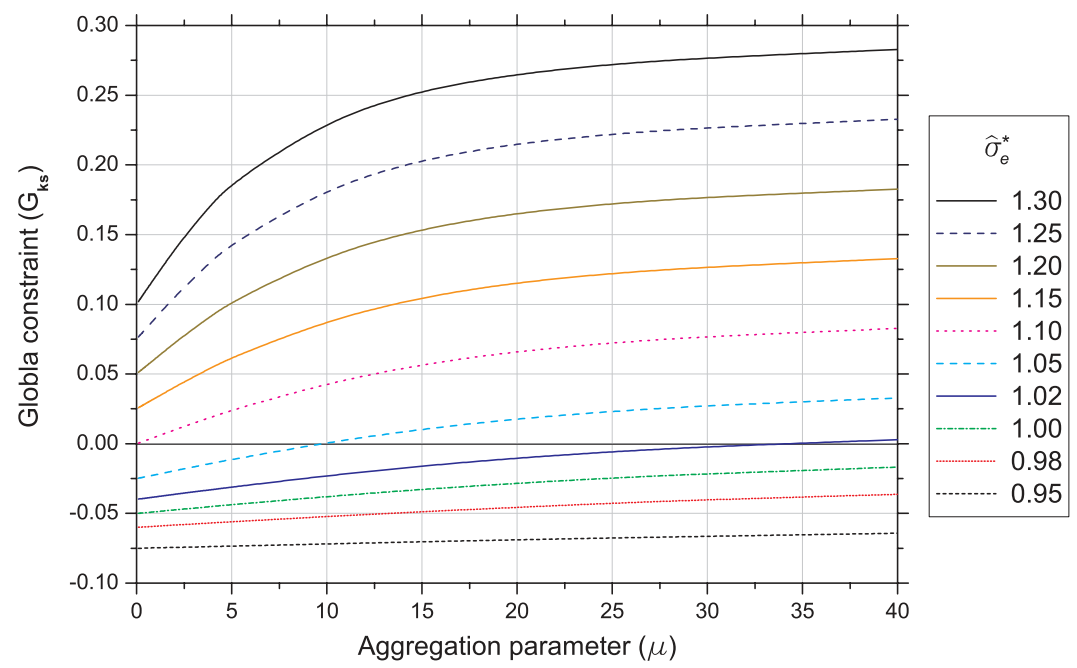

Figure 1: Global constraint versus $\mu$ for a growing value of the stress.

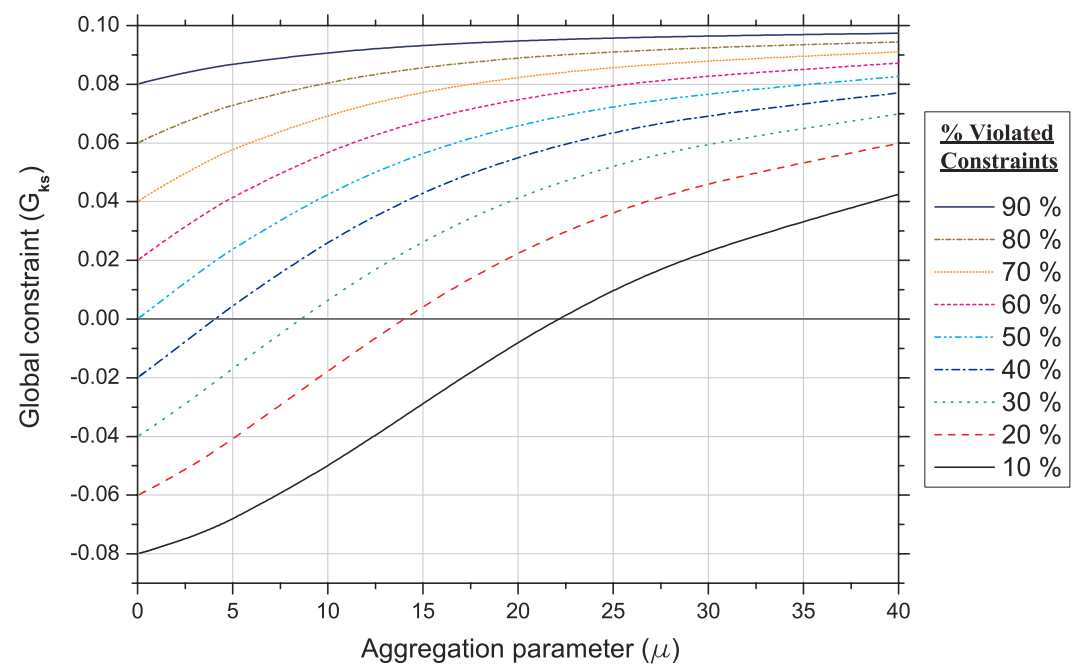

Figure 2: Global constraint versus $\mu$ for a growing $\%$ of violated local constraints. 


\section{Optimization algorithms}

In practice, the local approach leads to mathematical programming problems type (5) with a large number of highly non-linear constraints type (6). An improved SLP algorithm with quadratic line-search seems to be the right choice to solve this kind of problems [8]. Thus, the linear approximation to problem (5) is stated (with additional side constraints) and solved at each iteration by means of the Simplex method [9]. The inactive constraints are disregarded, with the aim of saving computational resources. Even though the obtained results are quite promising [4], both the data storage and the computing time associated to stating and solving the underlying linear programming problems grow very fast with the number of elements $N_{e}$. This fact severely restricts the applicability of the technique.

On the other hand, the global constraints approach leads to mathematical programming problems type (7) with only one highly non-linear constraint type (8). To solve this kind of problems we propose the modified inverse barrier function

$$
\phi(\boldsymbol{\rho}, r)=F(\boldsymbol{\rho})\left[1-r \frac{1}{G_{K S}(\boldsymbol{\rho})}\right] .
$$

In comparison with the standard definition [10], the inverse of the global constraint in the above expression is multiplied times the objective function. We recall that the expression of $G_{K S}(\boldsymbol{\rho})$ type (8) is non-dimensional, unlike the expression of $F(\boldsymbol{\rho})$ type (2). The rescaling introduced by this product improves the numerical conditioning of the problem, while possible dimension conflicts are prevented. Furthermore, it helps to adequately calibrate the value of the so-called barrier parameter $r$. Then, the quasi-unconstrained non-linear programming problem

$$
\begin{array}{lll}
\text { Find } & \boldsymbol{\rho}=\left\{\boldsymbol{\rho}_{e}\right\}, & e=1, \ldots, N_{e} \\
\text { that minimizes } & \phi(\boldsymbol{\rho}, r), & \\
\text { verifying } & 0<\rho_{\text {min }} \leq \rho_{e} \leq 1, & e=1, \ldots, N_{e}
\end{array}
$$

is solved by means of the Fletcher-Reeves conjugate gradient method [10], which performance is improved by using a complementary quadratic line-search. On a regular basis, both the data storage and the computing time associated to stating and solving the underlying quasi-unconstrained non-linear programming problems grow linearly with the number of elements $N_{e}$. This fact expands the applicability of the technique far beyond the possibilities of the local approach.

Both approaches require the full first order sensitivity analysis at each iteration. This is done by means of an analytical implementation of the adjoint state method [11]. In this way we avoid storing a large amount of intermediate results while the computing effort devoted to solving linear systems is minimized. Both approaches also require one additional second order directional sensitivity analysis at each iteration. This is done by means of an analytical implementation of the direct differentiation method [11], since data storing and computing time are not critical issues at this point. The computational cost of the sensitivity analysis is indeed expected to be much lower (or even negligible) in the global approach. 


\section{Numerical examples}

Actually, the examples presented below are two-dimensional. The solutions are represented as 3D solids in order to facilitate the understanding of the results, being the false thickness proportional to the relative density at each point.

The first example corresponds to an arch-type structure with fixed supports. Figure 3 shows the dimensions of the domain and the position of the external load. Self-weight is also considered. The rectangular domain ( $1 \mathrm{~m}$ thick) containing the structure is discretized in $76 \times 38=2888$ eight-node quadrilateral elements. The concentrated load is distributed within three contiguous elements. The material being used is steel with density $\gamma_{\text {mat }}=7650 \mathrm{~kg} / \mathrm{m}^{3}$, Young's modulus $E=2.110^{5} \mathrm{MPa}$, Poisson's ratio $\nu=0.3$ and elastic limit $\widehat{\sigma}_{\max }=230 \mathrm{MPa}$.

Figures 4 and 5 compare the solutions obtained by means of the local ( $p=10$, $\varepsilon=0.01, q=1)$ and of the global approach $(p=10, \varepsilon=0.01, \mu=20)$.

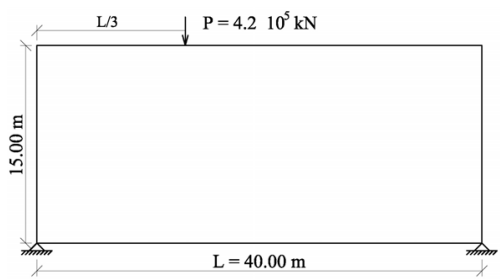

Figure 3: Example 1. Domain definition and external loads.
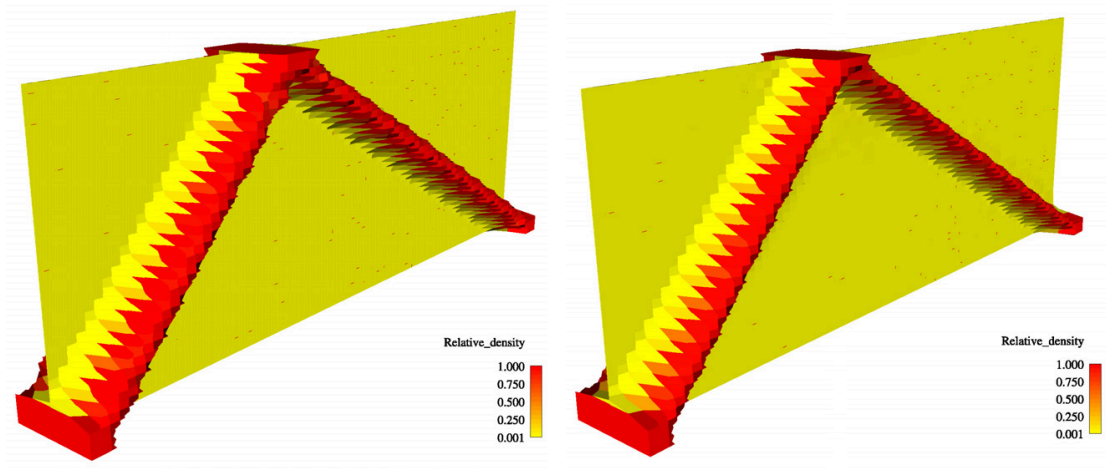

Figure 4: Example 1. Distribution of material at the final solution. (Local approach (left) vs. global approach (right).)

The second example corresponds to a classic MBB-type beam with sliding supports. Only half of the structure is analyzed, because of symmetry. Figure 6 shows the dimensions of the domain and the position of the external load. Self-weight is also considered. The rectangular domain ( $1 \mathrm{~m}$ thick) containing the structure 

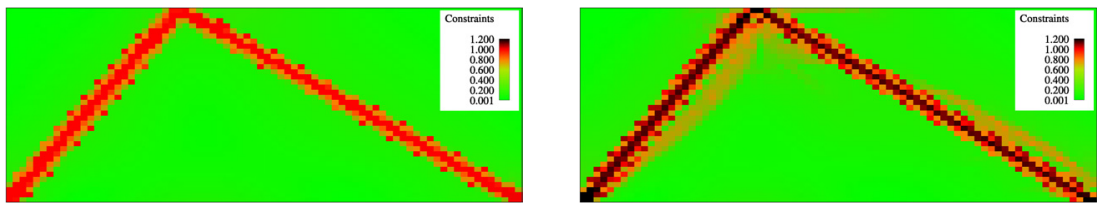

Figure 5: Example 1. Normalized reference stress $\left(\widehat{\sigma}_{e}^{*}\right)$ at the final solution. (Local approach (left) vs. global approach (right).)

is discretized in $60 \times 20=1200$ eight-node quadrilateral elements. The material being used is the same as in the former example.

Figures 7 and 8 compare the solutions obtained by means of the local ( $p=4$, $\varepsilon=0.02, q=1)$ and of the global approach $(p=4, \varepsilon=0.02, \mu=20)$.

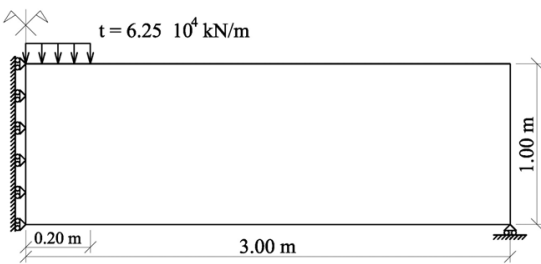

Figure 6: Example 2. Domain definition and external loads.
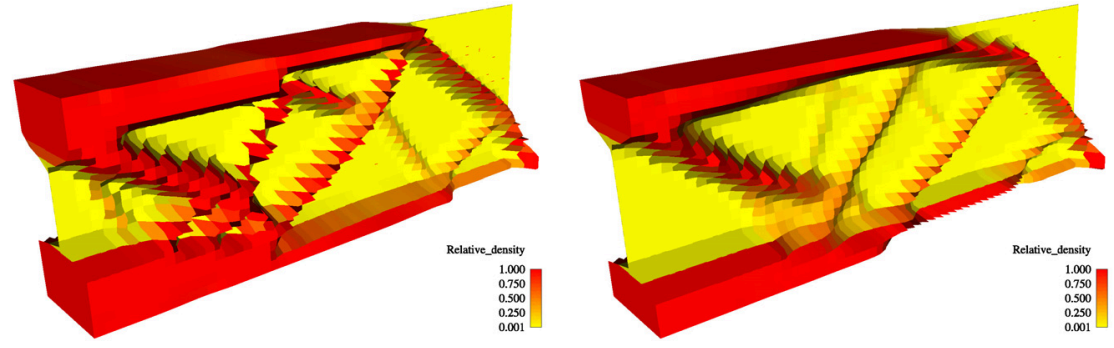

Figure 7: Example 2. Distribution of material at the final solution. (Local approach (left) vs. global approach (right).)
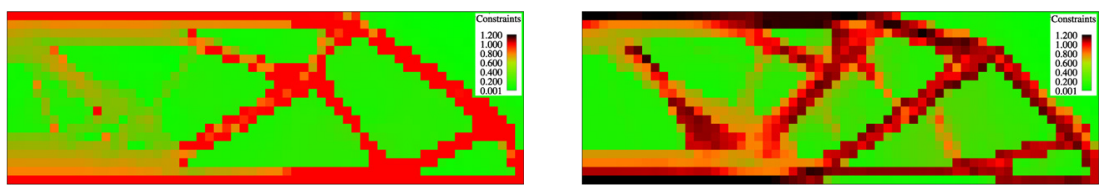

Figure 8: Example 2. Normalized reference stress $\left(\widehat{\sigma}_{e}^{*}\right)$ at the final solution. (Local approach (left) vs. global approach (right).) 


\section{Conclusions}

In this paper, we have proposed specific procedures for correctly stating local constraints (local approach) and for constraint aggregation (global approach) in structural topology optimization problems. We have also compared the performance of both types of constraints (global vs. local) in some application examples. The computational requirements (both the data storage and the computing time) have been an order of magnitude lower in the case of the global approach, as it was expected. In return, the results have not been exactly equivalent, but quite similar.

The impressive reduction in the computational cost due to the constraint aggregation clearly compensates for the slight loss of accuracy in the results. Moreover, the applicability of the technique is expanded far beyond its original possibilities.

\section{Acknowledgements}

This work has been partially supported by Grant Numbers DPI-2002-00297, DPI2004-05156 and DPI-2006-15275 of the "Ministerio de Educación y Ciencia" by Grant Numbers PGIDIT03-PXIC118001PN and PGIDIT03-PXIC118002PN of the "Dirección Xeral de $I+D$ " and by research fellowships of the "Universidad de A Coruña" and of the "Fundación de la Ingeniería Civil de Galicia".

\section{References}

[1] Bendsøe, M.P., Optimization of structural topology, shape, and material, Springer-Verlag: Heidelberg, 1995.

[2] Ramm, E., Schwarz S. \& Kemmler, R., Advances in structural optimization including nonlinear mechanics, Proc. of the European Congress on Computational Methods in Applied Sciences and Engineering (ECCOMAS 2000) ECCOMAS: Barcelona, 2000.

[3] Duysinx P. \& Bendsøe M. P., Topology optimization of continuum structures with local stress constraints, International Journal for Numerical Methods in Engineering, 43, pp. 1453-1478, 1998.

[4] Navarrina F., Muíños I., Colominas I. \& Casteleiro M., Topology optimization of structures: a minimum weight approach with stress constraints, $A d$ vances in Engineering Software, 36, pp. 599-606, 2005.

[5] Martins J. R. R. A. \& Poon N. M. K., On structural optimization using constraint aggregation, Proceedings of the VI World Congress of Structural and Multidisciplinary Optimization WCSMO6, ISSMO: Rio de Janeiro, 2005.

[6] Cheng G. D. \& Jiang Z., Study on topology optimization with stress constraints, Engineering Optimization, 20, pp. 129-148, 1992.

[7] Cheng G. D. \& Guo X., $\varepsilon$-relaxed approach in structural topology optimization, Structural Optimization, 13, pp. 258-266, 1997.

[8] Navarrina, F., Tarrech, R., Colominas, I., Mosqueira, G., Gómez-Calviño J. \& Casteleiro, M., An efficient MP algorithm for structural shape optimization problems, Computer Aided Optimum Design of Structures VII, eds. 
S. Hernández \& C.A. Brebbia, WIT Press: Southampton, pp. 247-256, 2001. [9] Dantzig G. B. \& Thapa M. N., Linear Programming I: Introduction, Springer-Verlag: New York, 1997.

[10] Fletcher R., Practical Methods of Optimization, John Wiley \& Sons: Chichester, 2000.

[11] Navarrina, F., López, S., Colominas, I., Bendito E. \& Casteleiro, M., High order shape design sensitivity: A unified approach, Computer Methods in Applied Mechanics and Engineering, 188, pp. 681-696, 2000. 